\title{
LIFTING VECTOR-VALUED MEROMORPHIC FUNCTIONS IN INFINITE DIMENSIONS
}

\author{
NGUYEN VAN KHUE AND NGUYEN THU NGA
}

(Communicated by Clifford J. Earle, Jr.)

\begin{abstract}
It is shown that the lifting problem for Fréchet-valued meromorphic functions on open subsets of a (DFN)-space has a solution.
\end{abstract}

Lifting holomorphic functions in infinite dimensions has been investigated by some authors. The problem for vector-valued meromorphic functions on complex manifolds was studied in [6]. The aim of this paper is to prove that the lifting problem has a solution for Fréchet-valued meromorphic functions on open subsets of a (DFN)-space.

\section{Preliminaries}

We shall use the standard notation from the theory of locally convex spaces as presented in the books of Pietsch [7] and Schaefer [8]. All locally convex spaces are assumed to be complex vector spaces and Hausdorff.

For a locally convex space $E$, we denote by $\mathscr{U}(E)$ the set consisting of all balanced convex neighbourhoods of zero in $E$. Let $U \in \mathscr{U}(E)$ and $p_{U}$ denote the Minkowski functional on $E$ associated to $U$. Then $E_{U}$ denotes the completion of the canonical normed space $E / \operatorname{Ker} p_{U}$. The canonical map from $E$ into $E_{U}$ is written by $\pi_{U}$.

\section{HOLOMORPHIC AND MEROMORPHIC FUNCTIONS}

Let $E$ and $F$ be locally convex spaces and $D \subseteq E$ be open. $A$ map $f: D \rightarrow F$ is called holomorphic if $f$ is continuous and $f \mid D \cap V$ is holomorphic for every finite dimensional subspace $V$ of $E$.

Now a holomorphic function $f: D_{0} \rightarrow F$, where $D_{0}$ is a dense open subset of $D$, is said to be meromorphic on $D$ if for every $z \in D$ there exists a neighbourhood $U$ of $z$ in $D$ and holomorphic functions

$$
g: U \rightarrow F, \quad \sigma: U \rightarrow \mathbb{C}
$$

such that

$$
f\left|U \cap D_{0}=g / \sigma\right| U \cap D_{0} \quad \text { with } \sigma \neq 0 .
$$

Received by the editors March 4, 1991 and, in revised form, October 13, 1992.

1991 Mathematics Subject Classification. Primary 46G20. 
Theorem 1. Let $S$ be a continuous linear map of a Fréchet space $E$ onto a Fréchet space $F$ and let $D$ be an open subset of a (DFN)-space $P$. Then for every $F$-valued meromorphic $f$ on $D$ there exists an E-valued meromorphic function $g$ on $D$ such that $S g=f$.

To prove the theorem we need the following.

Lemma 2. Every holomorphic function on an open subset of a (DFN)-space with values in a Fréchet space $F$ can be factorized through a compact map from a Banach space into $F$.

Proof. Consider an open set $D$ in a (DFN)-space $P$ and a holomorphic function $f$ on $D$ with values in $F$.

(i) Let $z \in D$. Since $D$ is $\sigma$-compact [5], it can be exhausted by an increasing sequence of compact sets $\left\{K_{n}\right\}$, with $z \in K_{1}$. Let $\left\{V_{n}\right\}$ be a decreasing neighbourhood basis of zero in $F$. For each $n \geq 1$ there exists $U_{n} \in \mathscr{U}(P)$ and $d_{n}>0$ such that

$$
K_{n}+U_{n} \subseteq D \text { and } f\left(K_{n}+U_{n}\right) \subset d_{n} V_{n} .
$$

Set

$$
U=\bigcap_{n \geq 1}\left(K_{n}+U_{n}\right)
$$

Since

$$
K_{n} \cap U=\bigcap_{1 \leq k \leq n}\left(K_{k}+U_{k}\right) \cap K_{n}
$$

and since $\bigcap_{1 \leq k \leq n}\left(K_{k}+U_{k}\right)$ is a neighbourhood of $z$ in $P$, it follows that $K_{n} \cap U$ is a neighbourhood of $z$ in $K_{n}$ for every $n \geq 1$. On the other hand, since $D$ is a $k$-space [5], $U$ is a neighbourhood of $z$ in $D$. From the inclusion

$$
f(U) \subseteq f\left(K_{n}+U_{n}\right) \subseteq d_{n} V_{n} \text { for every } n \geq 1
$$

we obtain the boundedness of $f(U)$.

Consider the Taylor expansion of $f$ at $z$ :

$$
f(z+h)=\sum_{n \geq 0} P_{n} f(z)(h),
$$

where

$$
P_{n} f(z)(h)=\frac{1}{2 \pi i} \int_{|\lambda|=2} f(z+\lambda h) / \lambda^{n+1} d \lambda
$$

for $h \in V, V \in \mathscr{U}(P), z+2 V \subseteq U$. Set

$$
B=\overline{\text { conv }} \bigcup_{n \geq 0} P_{n} f(z)(V) \text {. }
$$

Then $B$ is a balanced convex closed set in $F$ and $f$ induces a holomorphic function on $\pi_{V}(z+V)$ with values in the canonical Banach space $F(B)$ spanned by $B$.

(ii) By (i) we can find a countable open cover of $D,\left\{\tilde{U}_{i}=z_{i}+U_{i}\right\}, U_{i} \in$ $\mathscr{U}(P)$, a sequence of balanced convex bounded closed sets in $F,\left\{B_{i}\right\}$, and a sequence of holomorphic functions $f_{i}: \pi_{U_{i}}\left(\widetilde{U}_{i}\right) \rightarrow F\left(B_{i}\right)$ such that

$$
f_{i} \pi_{U_{i}}=f \mid \widetilde{U}_{i} \quad \text { for every } i \geq 1 .
$$


Take two sequences $\left\{\lambda_{i}\right\} \downarrow 0$ and $\left\{\mu_{i}\right\} \uparrow \infty$ such that

$$
B=\overline{\text { conv }} \bigcup_{i \geq 1} \lambda_{i} B_{i} \text { is bounded in } F
$$

and

$$
U=\bigcap_{i \geq 1} \mu_{i} U_{i} \in \mathscr{U}(P) .
$$

Such sequences exist by [4] for $\left\{\lambda_{i}\right\}$ and [5] for $\left\{\mu_{i}\right\}$. Since the canonical map from $F\left(\lambda_{i} B_{i}\right)$ into $F(B)$ is continuous for every $i \geq 1$, and $\left\{\pi_{U}\left(\widetilde{U}_{i}\right)\right\}$ is an open cover of $\pi_{U}(D)$ in $P / \operatorname{Ker} p_{U}$, it follows that the sequence $\left\{f_{i}\right\}$ defines an $F(B)$-valued holomorphic function $g$ on $\pi_{U}(D)$ with $g \pi_{U}=f$.

(iii) Let $\hat{g}$ be a holomorphic extension of $g$ to a neighbourhood $\widehat{D}_{U}$ of $\pi_{U}(D)$ in $P_{U}$. Take $V \in \mathscr{U}(P), V \subseteq U$ such that the canonical map $\pi_{V, U}: P_{V}$ $\rightarrow P_{U}$ is compact. Cover $\widehat{D}_{V}=\pi_{V, U}^{-1}\left(\widehat{D}_{U}\right)$ by a sequence of bounded open sets in $P_{V},\left\{W_{i}\right\}$, such that $\pi_{V, U}\left(W_{i}\right)$ is relatively compact in $\widehat{D}_{U}$ for every $i \geq 1$. Then $A_{i}=g \pi_{V, U}\left(W_{i}\right)$ for every $i \geq 1$ is relatively compact in $F(B)$. Take again a sequence $\left\{\alpha_{i}\right\} \downarrow 0$ such that

$$
A=\overline{\operatorname{conv}} \bigcup_{i \geq 1} \alpha_{i} A_{i}
$$

is compact in $F(B)$. It is easy to see that $\hat{g} \pi_{V, U}: \widehat{D}_{V} \rightarrow F(A)$ is holomorphic. Hence $f$ can be factorized through the compact map $F(A) \rightarrow F$. The lemma is thus proved.

\section{Proof of Theorem 1}

Cover $D$ by a sequence of open subsets $\left\{\widetilde{U}_{i}\right\}$ of $D$ such that $f \mid \widetilde{U}_{i}$ can be written in the form $h_{i} / \sigma_{i}$, where $h_{i}$ and $\sigma_{i}$ are holomorphic functions on $\widetilde{U}_{i}$ with values in $F$ and $\mathbb{C}$, respectively. By Lemma 2 , for each $i \geq 1$ we can find $U_{i} \in \mathscr{U}(P)$ and $B_{i}$, a balanced convex compact set in $F$ such that $h_{i}$ and $\sigma_{i}$ are factorized through $\pi_{U_{i}}: \widetilde{U}_{i} \rightarrow \pi_{U_{i}}\left(\widetilde{U}_{i}\right)$ and $F\left(B_{i}\right) \rightarrow F$. Take two sequences $\left\{\lambda_{i}\right\} \downarrow 0$ and $\left\{\mu_{i}\right\} \uparrow \infty$ such that

$$
B=\overline{\operatorname{conv}} \bigcup_{i \geq 1} \lambda_{i} B_{i} \quad \text { is compact in } F
$$

and

$$
U=\bigcap_{i \geq 1} \mu_{i} U_{i} \in \mathscr{U}(P)
$$

This implies that the two sequences $\left\{h_{i}\right\}$ and $\left\{\sigma_{i}\right\}$ define a meromorphic function $g$ on a neighbourhood $\widehat{D}_{U}$ of $\pi_{U}(D)$ in $P_{U}$ with $f=g \pi_{U}$. By [1] there exists a balanced convex compact set $A$ in $E$ such that $S(A)=B$.

Cover $\widehat{D}_{U}$ by a sequence of open sets $\left\{W_{j}\right\}$ in $\widehat{D}_{U}$ such that for each $j \geq 1$ there exist bounded holomorphic functions $g_{j}$ and $\sigma_{j}$ on $W_{j}$ with values in $F(B)$ and $\mathbb{C}$, respectively, with $g \mid W_{j}=g_{j} / \sigma_{j}, \sigma_{j} \neq 0$. Let $V \in \mathscr{U}(P)$, $V \subseteq U$ such that $T=\pi_{V, U}$ is nuclear. Thus $T$ can be written in the form

$$
T(x)=\sum_{k \geq 1} \lambda_{k}(x) e_{k}
$$


with $a=\sum_{k \geq 1}\left\|\lambda_{k}\right\|\left\|e_{k}\right\|<\infty$. Fix an index $j$. For each $x \in T^{-1}\left(W_{j}\right)$, set $2 r_{j, x}=p_{U}\left(T \bar{x}, \partial W_{j}\right)>0$. Consider the Taylor expansion of $g_{j}$ at $T(x)$ :

$$
g_{j}(T x+z)=\sum_{n \geq 0} P_{n} g_{j}(T x)(z)
$$

for $\|z\|<2 r_{j, x}, z \in P_{U}$, where

$$
P_{n} g_{j}(T x)(z)=\frac{1}{2 \pi i} \int_{|\lambda|=r_{j, x}} g_{j}(T x+\lambda z) / \lambda^{n+1} d \lambda
$$

for $\|z\| \leq 1, z \in P_{U}$. We have

$$
\left\|P_{n} g_{j}(T x)\right\| \leq M_{j} /\left(r_{j, x}\right)^{n} \quad \text { with } M_{j}=\sup \left\{\left\|g_{j}(z)\right\|: z \in W_{j}\right\} .
$$

Therefore

$$
\begin{gathered}
\sum_{n \geq 0} \sum_{k_{1}, \ldots, k_{n} \geq 1}\left(\delta_{j, x}\right)^{n}\left\|\lambda_{k_{1}}\right\|\left\|e_{k_{1}}\right\| \cdots\left\|\lambda_{k_{n}}\right\|\left\|e_{k_{n}}\right\| \\
\times\left\|P_{n} g_{j}(T x)\left(e_{k_{1}} /\left\|e_{k_{1}}\right\|, \ldots, e_{k_{n}} /\left\|e_{k_{n}}\right\|\right)\right\| \\
\leq M_{j} \sum_{n \geq 0}(1 / n !)\left(\delta_{j, x} n / r_{j, x}\right)^{n}\left(\sum_{k \geq 1}\left\|\lambda_{k}\right\|\left\|e_{k}\right\|\right)^{n} \\
=M_{j} \sum_{n \geq 0}(1 / n !)\left(\delta_{j, x} n a / r_{j, x}\right)^{n}<\infty \quad \text { with } \delta_{j, x}=r_{j, x} / 2 a e .
\end{gathered}
$$

It follows that $g_{j} T \mid x+\delta_{j, x} B_{V}$, where $B_{V}$ is the unit ball in $P_{V}$, can be written in the form

$$
g_{j} T(z)=\sum_{\alpha \in \mathscr{B}} \lambda_{\alpha}(z-x) a_{\alpha}^{j, x}
$$

for $z \in P_{V},\|z-x\|<\delta_{j, x}$, where

$$
\begin{aligned}
& \mathscr{B}=\left\{\alpha \in\left(\mathbb{Z}^{+}\right)^{\mathbb{N}}: \alpha_{j} \neq 0 \text { for only finitely many } j \in \mathbb{N}\right\}, \\
& \lambda_{\alpha}=\lambda_{\alpha_{1}} \cdots \lambda_{\alpha_{n}}, a_{\alpha}^{j, x}=P_{n} g_{j}(T x)\left(e_{\alpha_{1}}, \ldots, e_{\alpha_{n}}\right), \\
& \alpha=\left(\alpha_{1}, \ldots, \alpha_{n}, 0, \ldots,\right),
\end{aligned}
$$

and

$$
\sum_{n \geq 0}\left(\delta_{j, x}\right)^{n}\left\|\lambda_{\alpha}\right\|\left\|a_{\alpha}^{j, x}\right\|<\infty .
$$

Thus there exist $x_{j, k} \in T^{-1}\left(W_{j}\right), \delta_{j, k}>0, j, k=1,2, \ldots$, such that $\left\{x_{j, k}+\frac{1}{2} \delta_{j, k} B_{V}\right\}$ is an open cover of $T^{-1}\left(W_{j}\right)$ and

$$
\sum_{n \geq 0}\left(\delta_{j, k}\right)^{n}\left\|\lambda_{\alpha}\right\|\left\|a_{\alpha}^{j, k}\right\|<\infty, \quad a_{\alpha}^{j, k}=a_{\alpha}^{j, x_{j, k}}
$$

with $\left(g_{j} T\right)(x)=\sum_{\alpha \in \mathscr{B}} \lambda_{\alpha}\left(x-x_{j, k}\right) a_{\alpha}^{j, k}$ for $x \in x_{j, k}+\delta_{j, k} B_{V}$.

For each $n \geq 1$, set $A_{n}=\left\{\alpha \in \mathscr{B}: \max \alpha_{j} \leq n\right\}$ and

$$
S_{n}^{j, k}(x)=\sum_{\alpha \in A_{n}} \lambda_{\alpha}\left(x-x_{j, k}\right) a_{\alpha}^{j, k}
$$

for $x \in x_{j, k}+\delta_{j, k} B_{V}$. 
Let $\varepsilon>0$ be given. Take $m \in \mathbb{N}$ such that

$$
\sum_{i>m}\left\|\lambda_{i}\right\| i \mid e_{i} \|<\varepsilon
$$

We have

$$
\begin{aligned}
& \left\|\sum_{\alpha \in \mathscr{B}} \lambda_{\alpha}\left(x-x_{j, k}\right) a^{j, k}-S_{m}^{j, k}(x)\right\| \\
& \quad \leq \sum_{\max \alpha_{j}>m, n \geq 0}\left(\delta_{j, k}\right)^{n}\left\|\lambda_{\alpha_{1}}\right\| \cdots\left\|\lambda_{\alpha_{n}}\right\|\left\|a_{\alpha}^{j, k}\right\| \\
& \quad \leq\left(M_{j} \varepsilon / a\right) \sum_{n \geq 0}\left(\delta_{j, k} n / r_{j, x_{j, k}}\right)^{n}(1 / n !)\left(\sum_{i \geq 1}\left\|\lambda_{i}\right\|\left\|e_{i}\right\|\right)^{n} \\
& \quad=\left(M_{j} \varepsilon / a\right) \sum_{n \geq 0} 1 / n !(n / 2 a)^{n} .
\end{aligned}
$$

Thus $S_{n}^{j, k} \rightarrow g_{j} T$ uniformly on $x_{j, k}+\delta_{j, k} B_{V}$ for $j, k \geq 1$ as $n \rightarrow \infty$. Applying the method of Bishop [2] to the sequence $\left\{S_{n}^{j, k}\right\}, j, k \geq 1$, we can find a sequence of disjoint 1-dimensional projections $P_{q}^{n}$ in $F(B)$ such that

$$
S_{n}^{j, k}=\sum_{q \geq 1} P_{q}^{n} S_{n}^{j, k}=\sum_{q \geq 1} h_{q}^{n, j, k} v_{q}^{n}
$$

and

$$
\begin{gathered}
\left\|v_{q}^{n}\right\|=1, \quad\left\|P_{q}^{n}\right\| \leq 2^{\log _{2}^{2} 4 q}, \quad P_{q}^{n}\left(v_{q}^{n}\right)=v_{q}^{n} \\
\sup _{n \geq 1} \sum_{q \geq 1}\left\|h_{q}^{n, j, k}\right\|_{x_{j, k}+\delta_{j, k} B_{V}}<\infty \quad \text { for } j, k \geq 1,
\end{gathered}
$$

where $\tilde{\delta}_{j, k}=\frac{1}{2} \delta_{j, k}$ and $\left\|h_{q}^{n, j, k}\right\|_{x_{j, k}+\tilde{\delta}_{j, k} B_{V}}$ denotes the sup-norm of $h_{q}^{n, j, k}$ on $x_{j, k}+\tilde{\delta}_{j, k} B_{V}$.

Since $S(A)=B$, the map $S$ induces a continuous linear map $\widetilde{S}$ from $E(A)$ onto $F(B)$. Thus the open mapping theorem gives a constant $C>0$ such that for each $(n, q)$ there exists $u_{q}^{n} \in E(A)$ for which $\widetilde{S}\left(u_{q}^{n}\right)=v_{q}^{n}$ with $\left\|u_{q}^{n}\right\| \leq C\left\|v_{q}^{n}\right\|$ for $n, q \geq 1$.

Set

$$
\widetilde{S}_{n}^{j, k}(x)=\sum_{q \geq 1} h_{q}^{n, j, k} u_{q}^{n}
$$

for $x \in x_{j, k}+\tilde{\delta}_{j, k} B_{V}$. Then

$$
\begin{aligned}
& \sup _{n \geq 1}\left\{\left\|\tilde{S}_{n}^{j, k}(x)\right\|: x \in x_{j, k}+\tilde{\delta}_{j, k} B_{V}\right\} \\
& \quad \leq C \sup _{n \geq 1} \sum_{q \geq 1}\left\|h_{q}^{n, j, k}\right\|_{x_{j, k}+\tilde{\delta}_{j, k} B_{V}}<\infty .
\end{aligned}
$$

Thus the sequence $\left\{\widetilde{S}_{n}^{j, k}\right\}_{n \geq 1}$ is bounded in $\mathscr{O}\left(x_{j, k}+\tilde{\delta}_{j, k} B_{V}, E(A)\right)$, the space of holomorphic functions on $x_{j, k}+\tilde{\delta}_{j, k} B_{V}$ with values in $E(A)$ equipped with 
the compact-open topology. From the compactness of the canonical map $E(A) \rightarrow E$, we can assume that $\left\{\widetilde{S}_{n}^{j, k}\right\}_{n \geq 1}$ converges to $\widetilde{S}^{j, k}$ in $\mathscr{O}\left(x_{j, k}+\tilde{\delta}_{j, k} B_{V}, E\right)$ as $n \rightarrow \infty$ for all $j, k \geq 1$. Moreover we assume also that the sequences $\left\{v_{q}^{n}\right\},\left\{P_{q}^{n}\right\}$, and $\left\{h_{q}^{n, j, k}\right\}$ converge to $v_{q}, P_{q}$ and $h_{q}^{j, k}$ in $F, \operatorname{Hom}(F(B), F)$, the space of continuous linear maps from $F(B)$ into $F$, and $\mathscr{O}\left(x_{j, k}+\tilde{\delta}_{j, k} B_{V}\right)$, respectively, as $n \rightarrow \infty$ for all $j, k, q \geq 1$. On the other hand, from the relations

$$
P_{s}^{n} v_{q}^{n}=0 \quad \text { if } s \neq q \quad \text { and } \quad P_{s}^{n} v_{s}^{n}=v_{s}^{n}
$$

we have

$$
\begin{aligned}
\lim _{n \rightarrow \infty} & P_{s}^{n} \sigma_{j} g_{i} T=\lim _{n \rightarrow \infty} P_{s}^{n} \sigma_{j} s_{n}^{j, k} \\
\quad & =\lim _{n \rightarrow \infty} \sum_{q \geq 1} P_{s}^{n} \sigma_{j} h_{q}^{n, i, k} v_{q}^{n}=\lim _{n \rightarrow \infty} \sigma_{j} h_{s}^{n, i, k} v_{s}^{n}=\sigma_{j} h_{s}^{i, k} v_{s} .
\end{aligned}
$$

Similarly

$$
\lim _{n \rightarrow \infty} P_{s}^{n} \sigma_{i} g_{j} T=\lim _{n \rightarrow \infty} P_{s}^{n} \sigma_{i} S_{n}^{j, l}=\sigma_{i} h_{s}^{j, l} v_{s}
$$

Hence

$$
\sigma_{j} h_{s}^{i, k}=\sigma_{i} h_{s}^{j, l} \text { for all } i, j, k, l \geq 1 .
$$

This yields

$$
\sigma_{i} \widetilde{S}^{j, k}=\sigma_{j} \widetilde{S}^{i, l}
$$

on $\left(x_{j, k}+\tilde{\delta}_{j, k} B_{V}\right) \cap\left(x_{i, l}+\tilde{\delta}_{i, l} B_{V}\right)$ for all $i, j, k, l \geq 1$.

Thus the system $\left\{\widetilde{S}^{j, k} / \sigma_{j}\right\}$ defines an $E$-valued meromorphic function $g$ on $D$ such that $S g=f$. The theorem is proved.

\section{ACKNOWLEDGMENT}

The authors are grateful to the referee for comments and suggestions.

\section{REFERENCES}

1. R. G. Bartle and L. M. Graves, Mappings between function spaces, Trans. Amer. Math. Soc. 72 (1952), 400-413.

2. E. Bishop, Analytic functions with values in a Fréchet space, Pacific J. Math. 12 (1962), 1177-1192.

3. W. Gejler, Extending and lifting continuous linear mappings in topological vector spaces, Studia Math. 62 (1978), 295-303.

4. J. Horvath, Topological vector spaces and distributions. I, Addison-Wesley, Reading, MA, 1966.

5. J. Mujica, Domains of holomorphy in (DFC)-spaces, Functional Analysis Holomorphy and Approximation Theory, Lecture Notes in Math., vol. 843, Springer-Verlag, Berlin and New York, 1981, pp. 500-533.

6. N. V. Khue, On meromorphic functions with values in locally convex spaces, Studia Math. 73 (1982), 201-211.

7. A. Pietsch, Nuclear locally convex spaces, Akademie-Verlag, Berlin, 1972.

8. H. Schaefer, Topological vector spaces, Springer-Verlag, Berlin and New York, 1971.

Department of Mathematics, Pedagogical Institute, Hanol I, Hanol, Vietnam 